\title{
Inmersión e Interactividad Sin Fotorrealismo: Análisis de una posibilidad para modelos tridimensionales de proyectos arquitectónicos académicos
}

\begin{abstract}
Immersion, interactivity and Non-Photorealistic: Analysis of a possibility for three-dimensional models of academic architectural projects
\end{abstract}

- Felipe Etchegaray Heidrich

Universidade Federal de Pelotas, Brasil

felipeheidrich@gmail.com

- Ernest Redondo Dominguez

Universidad Politécnica de Cataluña, España

ernesto.redondo@upc.edu

\begin{abstract}
This paper had as objective to propose a development method for a graphical representations with immersion, interactivity and nonphotorealistic for use in the communication of academic architectural projects and which can be generated using the same digital models that students develop normally.
\end{abstract}

Keywords: Immersion, Interactivity, Non-Photorealistic, QTVR, Tablet

\section{Introducción}

Según comenta Sainz (2005), la representación del espacio arquitectónico se ve delimitada por las propiedades del medio gráficoen que se desenvuelve. Deestemodo, Lévy(1996)observa que "un texto impresoen papel, aunquegenerado por ordenador, no tiene estatus real o propiedad estética fundamentalmente distinta de un texto escrito con los instrumentos del siglo XIX" (p.41). Por lo tanto, los contenidos digitales, según Lévy (1996), solo tendrán una "nueva plasticidad" si estuvieran en otro medio que no el papel, o sea, con la visualización en la pantalla, y otros dispositivos interactivos.

En este sentido, mismo que el uso de representaciones digitales hagan parte del cotidiano de las escuelas de arquitectura, si observamos con atención, lo que aparentemente ocurre es que mismo con la utilización del medio digital, siegue el predominio de las representaciones en papel. Sin embargo, Lévy (1996) observa que considerar el medio digital como solo una herramienta a más para producir textos e imágenes en papel, corresponde a negar su riqueza propia, o sea, la interactividad.

A partir de esto, con objetivo primeramente de identificar la presencia o no de esta nueva plasticidad en contenidos digitales, el presente estudio hizo un análisis de las comunicaciones de proyectos de cuatro asignaturas de
Proyecto Arquitectónico en una escuela de Arquitectura en Brasil, la Faculdade de Arquitetura e Urbanismo (FAUrb) de la Universidade Federal de Pelotas (UFPel).

En este análisis de los ejercicios de proyecto, fueidentificada la utilización de contenidos digitales bidimensionales en 77,5\% de los proyectos y de modelos tridimensionales digitales en $69,3 \%$. Sin embargo, la inmersión y la interactividad no fueron identificadas en ninguno de los contenidos digitales utilizados por los alumnos en sus comunicaciones de proyectos. En una otra investigación con los estudiantes de la misma escuela se verificó, que para ellos, la ventaja en el uso de los contenidos digitales está principalmente en la posibilidad de obtención de imágenes fotorrealistas.

Todavía, Ching (1986) comenta que: "El propósito primario del grafismo arquitectónico es la comunicación" (p. 172). Según comenta el autor, los dibujos utilizados en una presentación arquitectónica son simplemente herramientas de comunicación. Además, como observa Montes Serrano (1992) "lo normal, lo habitual en el arte de representación, no es la ilusión, sino la información" (p. 19). De mismo modo, él aún destaca que tenemos de entender que la función de toda representación es la descripción.

En este sentido, así como Bloomer (1982) observa "cualquier arquitectura es un estímulo potencial del movimiento, sea este real o imaginado" (p.72), una información que deberíamos 
describir en las comunicaciones de proyecto es el movimiento, independientemente de este ser del observador o apenas en la dirección de visualización. Esta representación de movimiento asociada a la interacción y alguna posibilidad de inmersión puede ser un uso más apropiado del medio digital como demuestran algunos estudios.

Según el estudio de Hemmerling (2008) sobre la utilización de ambientes digitales inmersivos en la representación de espacios arquitectónicos, esta utilización intensifica la percepción del proyecto. Además, al navegar en tiempo real a través de una escena digital, la sensación de inmersión amplia la percepción significativamente, en comparación con las representaciones bidimensionales de proyecto. Así, según Hemmerling (2008) la percepción en los ambientes digitales inmersivos contribuye significativamente en el proceso de evaluación y decisión sobre el diseño espacial.

De acuerdo con Baltazar et al (2014) y su estudio, el cual analiza la utilización de un ambiente digital inmersivo de bajo costo, para la simulación de un proceso constructivo con manipulación de los componentes de construcción y sus parámetros, la utilización de los ambientes interactivos pueden incluso superar las cuestiones de visualización.

En este sentido, el estudio de Dorta (2007) propuso un sistema inmersivo para ideación de proyecto, en lo cual, partiendo de técnicas desarrolladas por el autor en estudios anteriores (Dorta, 2004; Dorta, 2005), el espacio de ideación híbrido permitía al diseñador utilizar técnicas tradicionales de dibujo para hacer modelos a su alrededor en tiempo real y en escala, utilizando una tableta digital y un dispositivo de proyección inmersiva, lo que, según el autor, proporciona sensación de inmersión y presencia.

Con estas informaciones previas de las posibilidades de utilización de interacción e inmersión, este estudio buscó identificar una posibilidad de generar representaciones con inmersión e interactividad, y que fuera posible a través de la utilización de contenidos ya habitualmente desarrollados por los estudiantes. De este modo, el presente estudio partió del uso de modelos digitales predominantemente desarrollados por los estudiantes de la escuela en que se hizo la investigación, o sea, modelos tridimensionales desarrollados en Sketchup. Además, el estudio tenía como objetivo utilizar una visualización de modelos digitales non-photorealistic, o mejor, sin fotorrealismo, que según Strothotte y Schlechtweg (2002) se trata de la generación de contenidos digitales que, en términos generales, parecen estar hecho "a mano".

\section{Desarrollo del Estudio}

El desarrollo del estudio empezó por la definición de una representación con las características deseadas, o sea, con posibilidad de inmersión e interactividad. En este sentido fue definida la utilización de una técnica en particular, que ofrece la posibilidad de realizar una experiencia de Realidad Virtual con un material informático sencillo, que es el QuickTime VR (QTVR).
Esta técnica, desarrollada por la Apple Computer en 1991, permite producir un sistema de realidad virtual de bajo costo, el cual no necesita de equipos especiales ni ordenadores muy potentes. Lo que este estudio consideró ideal para aplicación en una escuela pública con pocos recursos como la escuela donde se realizó este estudio.

Esta tecnología, elegida para utilización en el estudio, simula la presencia de un observador dentro de un espacio, mediante la manipulación de una secuencia de imágenes estáticas, donde el usuario, por medio de interacción con estas imágenes, cambia la dirección del punto de vista dentro del espacio posibilitando mover la dirección de visualización de la escena (Hippolyte, 1999).

Esta técnica de representación, a pesar de no ser una novedad, con la popularización de las tabletas, adquirió nuevas posibilidades de interacción e inmersión, o sea, lo que antes se tenía que hacer por medio de manipulación de las imágenes con el ratón, actualmente con la utilización de tabletas se hace con el movimiento del proprio equipamiento. Así, que este tipo de interacción acaba por transformar la tableta en una ventana de visualización, como si el observador estuviera dentro del modelo tridimensional, lo que amplía la sensación de inmersión e interactividad.

A partir de la identificación de la técnica a ser utilizada, el estudio partió para la definición de un método de desarrollo de este tipo de representación, teniendo como base un tipo de modelo digital desarrollado a menudo por los estudiantes de la escuela en que se hizo el estudio, o sea, un modelo tridimensional desarrollado en Sketchup. Así que se propuso un método de desarrollo con las siguientes etapas: definición del punto de visualización; desarrollo de las imágenes estáticas; montaje de la imagen QTVR; visualización inmersiva con tableta.

1) Definición del punto de visualización: por ser un tipo de representación basado en un punto estático que mira en todas las direcciones, y lo normal en los modelos tridimensionales desarrollado por los estudiantes es que no haya contenidos alrededor de la edificación representada, este tipo de técnica se torna más adecuada para la visualización de espacios internos de la edificación propuesta. Así, esta etapa consiste en elegir un punto en el interior del modelo y posicionar el observador en este punto, definiendo la altura y la dirección de la visualización inicial;

2) Desarrollo de las imágenes estáticas: con el punto de visualización definido, se propone la utilización de un plugin de uso libre para el software SketchUp, llamado Cubic Pano Out. Este plugin genera un grupo de seis imágenes las cuales representan la visualización de las seis caras de una caja con el centro en el punto de visualización elegido. En esta etapa de desarrollo es que se elige la apariencia sin fotorrealismo de la representación, para esto es suficiente elegir el "estilo" de apariencia deseada. En verdad, cualquier imagen generada exclusivamente con la utilización de las herramientas propias del SketchUp será sin fotorrealismo, pero, es posible, con la alteración del "estilo" de apariencia utilizado, definir 
si las imágenes serán más, o menos parecidas con dibujos hechos a mano;

3) Montaje de la imagen QTVR: a partir de las imágenes generadas, es necesario la conversión de ellas en un archivo específico de la tecnología QuickTime VR (QTVR) que es el archivo en formato *.MOV que contiene la interactividad deseada. Para esto, se propone la utilización del software de uso libre GoCubic, que a partir de la selección de la secuencia de las imágenes estáticas hace la conversión en una única imagen en formato QTVR;

4) Visualización inmersiva con tableta: la visualización del archivo *.MOV en un ordenador, que puede ser hecha con el software Quicktime, a pesar de tener interactividad, es considerado como un tipo de Realidad Virtual sin inmersión por tener su visualización en una pantalla y la interacción en el ratón. Con la utilización de tableta en la visualización del archivo QTVR ocurre que la sensación de inmersión es mejorada por tener la posibilidad de direccionar la visualización a través del posicionamiento de la tableta, como si esta fuera una ventana manipulable por los movimientos del observador, a través de la cual se hace la visualización del modelo digital. Para esto, el archivo QTVR necesita ser transferido para la tableta donde exista un aplicativo específico para su visualización. El aplicativo a ser utilizado puede variar conforme el sistema operacional de la tableta, si iOS o Android.

\section{Resultados}

Con el método de desarrollo definido, el estudio desarrolló ejemplos de la representación propuesta. Para esto, el estudio utilizó un modelo tridimensional hecho en Sketchup, de la Farnsworth House del arquitecto Mies van der Rohe. El modelo utilizado fue desarrollado a partir de una composición de cuatro modelos obtenidos de un banco de modelos tridimensionales digitales, denominado 3D Warehouse. Con este modelo, fueron seguidas las cuatro etapas propuestas por el método descrito para generar imágenes sin fotorrealismo en formato QTVR.

De este modo, en la definición del punto de visualización fueron utilizadas dos líneas para indicar el punto deseado, siendo una de las líneas la posición y la altura del punto de deseado, y la segunda línea la dirección y la altura de la visualización inicial, puntos 1 y 2 en la figura 1.

En seguida, para el desarrollo de las imágenes estáticas fue seleccionada la camera Cubic Pano Out, específica del plugin utilizado y generadas las seis caras de una caja (fig. 2) que tiene en su centro el punto de visualización elegido en la etapa anterior.

Con las imágenes estáticas listas, la etapa de montaje de la imagen QTVR consistió en la utilización del software GoCubic, donde fueron seleccionadas las imágenes estáticas y fue generado el archivo en formato *.MOV (fig. 03).

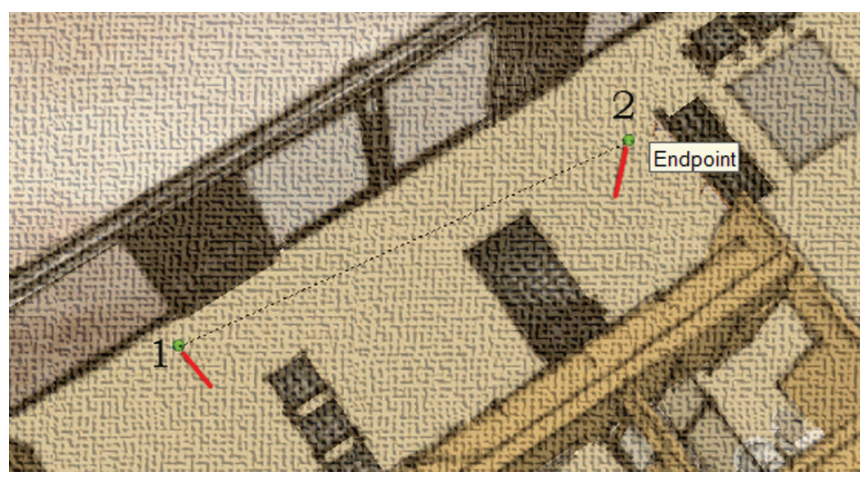

Figura 1: Indicación de los puntos utilizados para definir la localización del punto central de la representación.

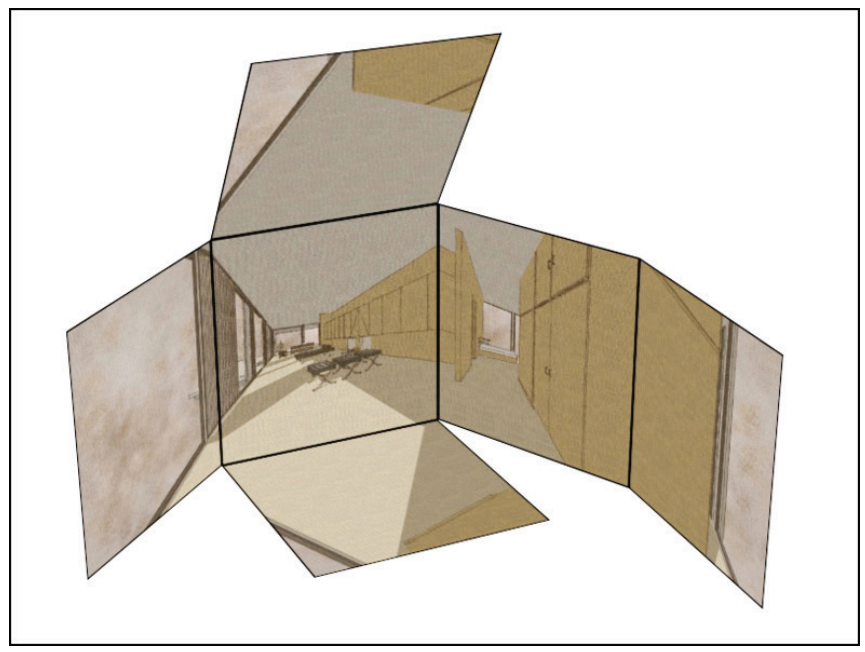

Figura 2: Imágenes estáticas con las seis caras de una caja, que tiene en su centro el punto de visualización elegido.

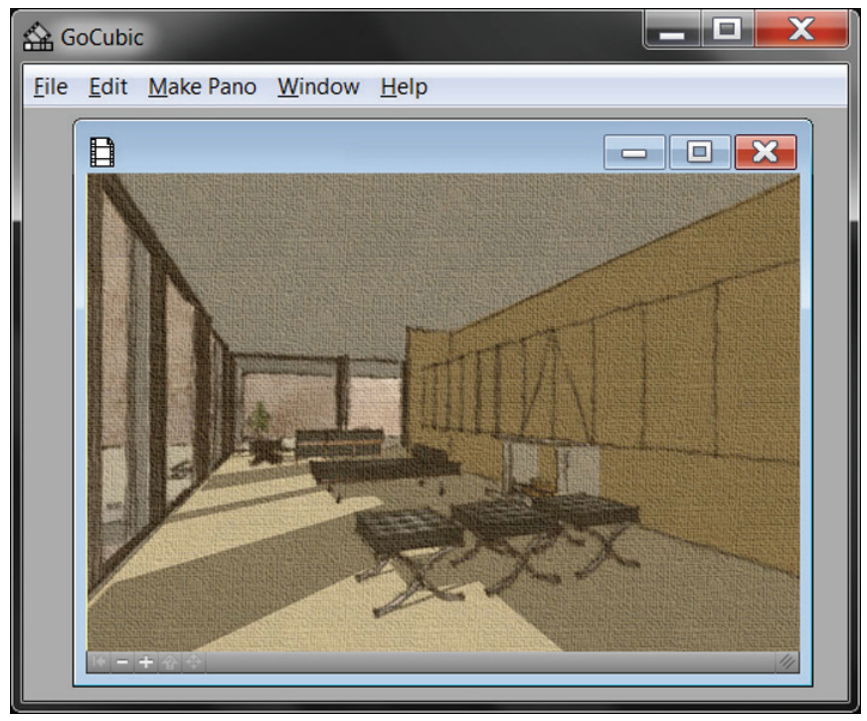

Figura 3: Software GoCubic y desarrollo del archivo QTVR.

Para la visualización inmersiva con tableta, el estudio utilizó un aplicativo para el sistema operacional iOS llamado QuickPano. Este aplicativo permite dos tipos de interacción: 
una definida como "sin uso del gyroscope", en la cual la interacción es hecha con la manipulación de la imagen a través de toques en la tela de la tableta; y otra definida como "con uso del gyroscope", en la cual la interacción es hecha con la manipulación de la tableta como si esta fuera una ventana de posición manipulable.

Después de desarrolladas y transferidas para una tableta, las imágenes en formato QTVR, fueron enseñadas para un grupo de noventa estudiantes, los cuales respondieron preguntas cuanto a la manipulación y el uso de este tipo de representaciones y cuanto a la ventaja de este tipo de representación.

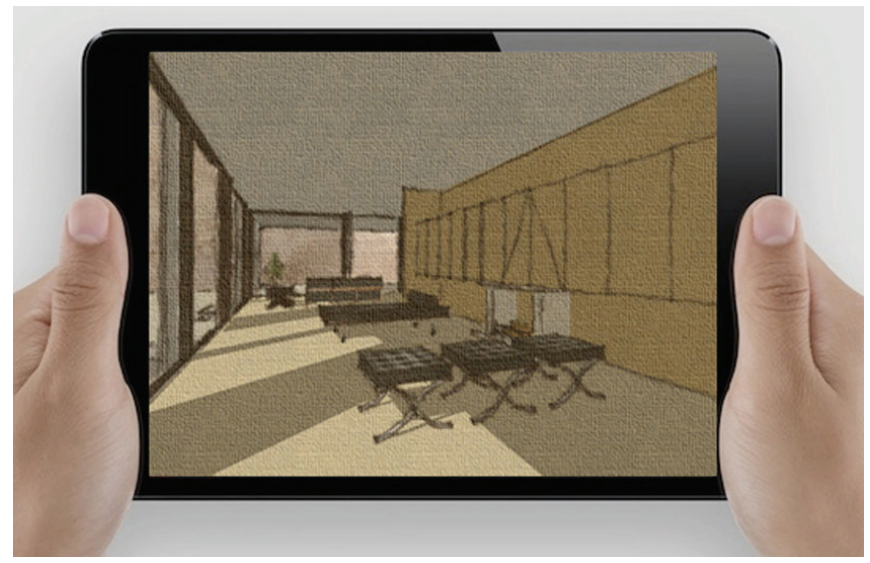

Figura 4: La imagen QTVR visualizada en una Tableta.

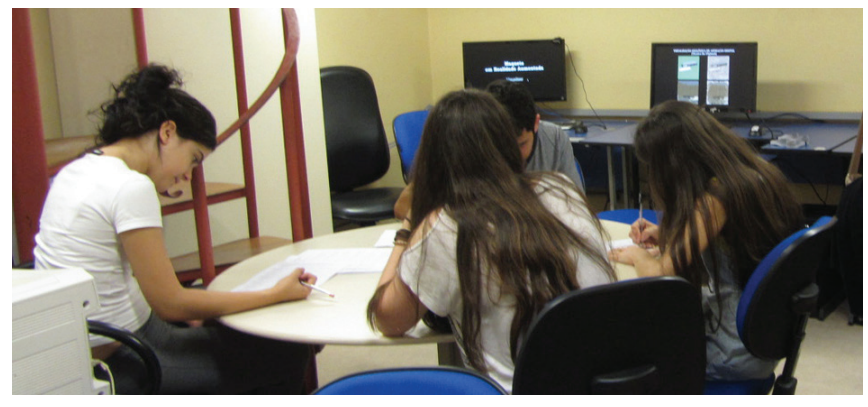

Figura 5: Foto de los estudiantes respondiendo las cuestiones propuestas.

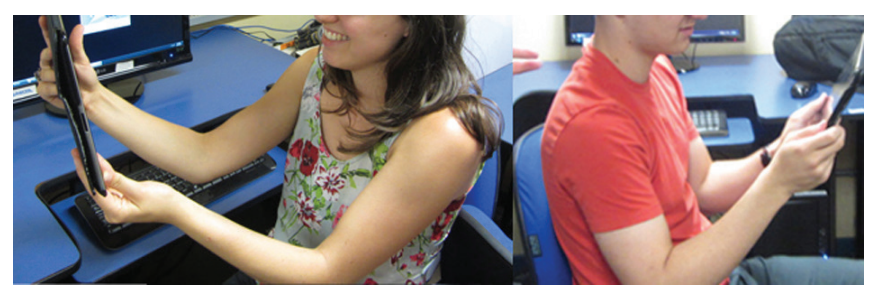

Figura 6: Foto de los estudiantes visualizando las representaciones con la técnica QTVR.

Con relación a la manipulación de las representaciones con la técnica QTVR en una tableta, las respuestas de los estudiantes demostraran que $88 \%$ de ellos la consideraron fácil y $12 \%$ la consideraron compleja, pero de fácil aprendizaje. Con relación a la utilización de este tipo de representación, todo el grupo la consideró útil y $68 \%$ dijeron que les gustarían utilizarla en sus comunicaciones de los ejercicios de proyecto. En las respuestas descriptivas, algunas de las respuestas obtenidas sobre la ventaja de la representación interactiva fueron: "Mejora la comprensión y visualización del proyecto"; "Amplía la percepción de los espacios"; "Acerca el proyecto de la realidad"; "Tiene la ventaja de estar prácticamente dentro del proyecto"; "Mejora la comprensión de como su proyecto será en la realidad".

\section{Conclusiones}

Este estudio tuvo como objetivo proponer un método de desarrollo para un tipo de representación con inmersión e interactividad, que, en verdad, es bastante sencillo y de fácil desarrollo, pero, a pesar de esto, no es utilizado en las comunicaciones de proyectos arquitectónicos en la escuela de arquitectura en que se hizo el estudio, mismo que casi $70 \%$ de los estudiantes tengan el elemento principal para el desarrollo de esta representación, los modelos tridimensionales de sus proyectos.

Además, la principal contribución del estudio consistió en verificar que mismo al utilizar imágenes sin fotorrealismo para enseñar las representaciones a los estudiantes, cuando estos describieron la ventaja de la representación enseñada, utilizaron la palabra 'realidad'. Lo que puede demostrar que la realidad de la representación de un espacio arquitectónico puede no estar en una apariencia realística de esta representación, y si, en la posibilidad de inmersión e interactividad con la información representada.

\section{Referencias}

Baltazar, Ana Paula; Souza, Emídio Dias Maciel e; Pontes, Mateus Moreira; Gonçalves, Filipe Silva; Metzker, Luiza Silva. (2014). Ambiente de imersão virtual como ferramenta para mudança de paradigma no processo de projeto arquitetônico: da representação à interação. In: Proceedings of the XVIII Conference of the Iberoamerican Society of Digital Graphics: Montevideo.

Bloomer, Kent C. (1982). Cuerpo, memoria y arquitectura. Título original: Body, Memory, and Architecture. Traducido por: María Teresa Muñoz Jiménez. Blume Ediciones: Madrid. Dorta, Tomas V. (2004). Drafted Virtual Reality: A new paradigm to design with computers. In Proceedings of the CAADRIA Conference: Seoul.

Dorta, Tomas V. (2005). Hybrid Modeling: Manual and digital media in the first steps of the design process. In Proceedings of the eCAADe Conference: Lisbon.

Dorta, Tomas V. (2007). Augmented Sketches and Models: The Hybrid Ideation Space as a Cognitive Artifact for Conceptual Design. Digital Thinking in Architecture, Civil Engineering, Archaeology, Urban Planning and Design: Montreal.

Hemmerling, Marco. (2008). Digital Material: Perception, 
Interaction and Immersion in Virtual Environments. In: Proceedings of the 9th DDSS-Conference: Eindhoven.

Hippolyte O., Pedro L. (1999). Visualización de edificaciones a través de la tecnología QTVR - Quick Time Virtual Reality. Actas de la 1ra Conferencia Venezolana sobre Aplicación de Computadores en Arquitectura. Caracas.

Lévy, Pierre. (1996). O que é o virtual? Titulo original: Qu'estce que Le virtuel? Tradução: Paulo Neves. São Paulo: Ed 34.
Montes Serrano, Carlos. (1992). Representación y Análisis Formal: Lecciones de análisis de forma. Universidad de Valladolid, Secretariado de Publicaciones: Valladolid.

Sainz, Jorge. (2005). El Dibujo de Arquitectura: Teoría e historia de un lenguaje gráfico. Editorial Reverté. Madrid. Strothotte, Thomas; Schlechtweg, Stefan. (2002). NonPhotorealistic Computer Graphics - Modeling, Rendering and Animation. Elsevir Science, San Francisco. 